\title{
TỔNG HợP LỚP FILM BÁN DẪN MẦM TINH THỂ ZNO TRÊN KÍNH DẪN TRONG SUỐT ITO VỚI THIẾT BỊ TỰ CHẾ SPIN - COATING
}

\author{
VĂN THANH KHUÊ, HUỲNH NGUYỄN VIẸTT ĐỨC, VÕ THÀNH CÔNG, NGUYỄN VĂN TRỌNG \\ Khoa Công nghệ Hoá học, Truờng Đại học Công nghiệp Thành phố Hồ Chí Minh \\ vanthanhkhue@iuh.edu.vn
}

\begin{abstract}
Thiết bị spin coater được thiết kế, chế tạo và ứng dụng để tổng hợp vật liệu màng mỏng đồng nhất trên chất nền phẳng với chi phí phù hợp, dễ sử dụng, khả năng hoạt động ở 2 pha tốc độ với khoảng tốc độ điều chỉnh là 300 - 4800 rpm, độ ổn định tốc độ cao và phù hợp với chất nền phẳng có nhiều hình dạng khác nhau với kích thước tối đa $10 \mathrm{~cm}$. Thiết bị được phát triển trên nền tảng Arduino và công nghệ in nhựa $3 \mathrm{D}$. Film bảng mỏng của vật liệu chất bán dẫn mầm tinh thể $\mathrm{ZnO}$ phủ trên đế kính dẫn trong suốt ITO có kích thước $25 \mathrm{~mm}$ x $25 \mathrm{~mm}$ được tổng hợp bằng thiết bị tự chế spin-coater và phương pháp nhiệt. Các phương pháp phân tích hóa lý hiện đại scanning electron microscopy và x-ray diffraction được áp dụng để nghiên cứu hình thái học và cấu trúc tinh thể của lớp vật liệu. Lớp mầm tinh thể $\mathrm{ZnO,} \mathrm{là} \mathrm{giai}$ đoạn quan trọng trước khi phát triển tinh thể với các hình thù khác nhau ứng dụng trong, điện cực quang điện, thiết bị điện tử quang điện hoá như màn hình cảm ứng, cảm biến, transistor, solar cell.
\end{abstract}

Từ khóa. Vật liệu bán dẫn, Zinc oxide, spin-coating, film bản mỏng, tinh thể.

\section{CONSTRUCTING A ZnO NUCLEI CRYSTAL SEMICONDUCTOR LAYER ONTO ITO GLASS WITH A CUSTOM-MADE SPIN-COATING EQUIPMENT}

\begin{abstract}
A custom-made spin coating equipment was designed and fabricated to apply in synthesizing the homogeneous thin films of nuclei materials with low cost and easy to use. The asfabricated equipment can work at the rate of from 300 to $4800 \mathrm{rpm}$. The equipment was built on Arduino technique and 3D printing technology. On the other hand, thin film of $\mathrm{ZnO}$ crystalline nuclei material was synthesized on an ITO glass with diameter $25 \mathrm{~mm}$ x $25 \mathrm{~mm}$ by using the asfabricated spin coater and annealing method. Modern physicochemical analytical techniques used to characterize the thin film material such as X-ray diffraction (XRD), scanning electron microscopy (SEM) resulting in the crystal structure and morphology of the film. The nuclei film of material is crucial stage of growing the material on a planar base.
\end{abstract}

Keywords: semiconducting materials, zinc oxide, spin-coating, thin film, crystal.

\section{GIỚI THIỆU}

Được nghiên cứu và sử dụng rộng rãi từ đầu thế kỷ 20 [1], phương pháp spin coating (phủ quay) đã được nhiều nhà khoa học trên thế giới sử dụng trong việc chế tạo các vật liệu, thiết bị hoặc linh kiện ở kích thước nhỏ (Micro-fabrication) [2] từ ngưỡng micromet [3] và có thể thu nhỏ đến kích thước nanomet [4]. Spin Coating bao gồm các ưu điểm như: vật liệu có độ đồng đều và tinh khiết cao, có thể điều khiển tính chất lớp màng dễ dàng bằng tốc độ quay, thời gian quay, gia tốc và khắc phục nhược điểm của các phương pháp khác: thiết bị sử dụng đơn giản, thao tác dễ dàng, nhanh chóng. Tuy nhiên, spin coating vẫn có nhược điểm: độ chọn lọc vật liệu phủ trên một khu vực nhất định khó thực hiện và phức tạp, không thể phủ màng trên những bề mặt nền tinh vi, phức tạp, gồ gề, chỉ có thể sử dụng trong môi trường phòng thí nghiệm nhỏ lẻ phục vụ nghiên cứu, phương pháp không có tính tự động hoá cao. 
Spin coating là phương pháp sử dụng lực ly tâm của đĩa đặt vật thể khi quay ở tốc độ cao (300 10.000 rpm) để phủ đều dung dịch có độ nhớt xác định trên bề mặt vật thể cần phủ, được gọi là chất nền, như kính polymer, kính thủy tinh dẫn điện trong suốt - ITO/FTO (indium tin oxide/fluorine doped tin oxide), tấm silicon,... với độ dày mong muốn, kết hợp với quá trình bay hơi dung môi để hình thành film vật liệu bản mỏng. Nguyên nhân là do trong quá trình phủ quay, sự tương tác giữa lực bám giữa bề mặt chung của dung dịch/chất nền và lực ly tâm tác động lên dung dịch, làm dung dịch có xu hướng tỏa tròn tạo thành một lớp phủ có độ dày nhỏ và đồng đều [5]. Quy trình phủ quay được thực hiện như sau: (1) Đầu tiên, dung dịch được rót một lượng dư trên bề mặt chất nền cần phủ, đĩa quay ở trạng thái đứng yên hoặc quay với vận tốc góc chậm, (2) đĩa quay được tăng tốc dần đến tốc độ mong muốn và duy trì trong khoảng thời gian nhất định. [6]

Tuy quy trình thực hiện phương pháp đơn giản, đã có nhiều bài báo lý thuyết và thực nghiệm được thực hiện nhằm giải thích sự phục thuộc các tính chất điện, quang, hình thái học của vật liệu vào các thông số quy trình spin coating $[7,8]$. Vì vậy, việc kiểm soát chính xác tốc độ quay, gia tốc quay và thời gian ảnh hưởng quan trọng đến tính chất vật liệu cần đạt được.

Vật liệu bán dẫn được nghiên cứu và ứng dụng trong nhiều lĩnh vực như xúc tác, điện tử, công nghệ sơn, $[9,10,11] \ldots$, Chất bán dẫn tinh thể $\mathrm{ZnO}$ có vùng năng lượng cấm $\mathrm{E}_{\mathrm{g}}=3.2 \mathrm{eV}$ [12] là vật liệu dễ tổng hợp, thân thiện với môi truòng được nghiên cứu phát triển với những hình thái học khác nhau nhằm nâng cao hiệu quả sử dụng, khắc phục được những nhược điểm hiện hữu của vật liệu. Cấu trúc $1 \mathrm{D}$ của vật liệu được phát triển để tăng khả năng hoạt tính quang hóa của vật liệu nhờ sự giảm thiểu sự tái kết hợp của

C 2020 Trường Đại học Công nghiệp thành phố Hồ Chí Minh cặp điện tử quang sinh $\left(\mathrm{e}^{-}, \mathrm{h}^{+}\right)$. $[12,13,14]$ Những nghiên cứu tổng hợp film bản mỏng cấu trúc $1 \mathrm{D}$ dưới dạng hình thái que thẳng hàng của vật liệu được quyết định bởi yếu tố quan trọng là lớp mầm tinh thể ban đầu của lớp film. Các que tinh thể có thể sắp xếp một cách thẳng hàng, mật độ phân bố cũng như kích thước đường kính của que chịu sự ảnh hưởng lớn của phương pháp tiền tổng hợp lớp mầm tinh thể ban đầu.

Trong nghiên cứu này, thiết bị spin coater được thiết kế và chế tạo áp dụng sử dụng nguồn nguyên linh kiện dễ dàng tìm kiếm, công nghệ chế tạo đơn giản và chi phí phù hợp ứng dụng vào lĩnh vực nghiên cứu tổng hợp vật liệu màng mỏng một cách chính xác, hiệu quả và có năng suất. Bên cạnh đó, lớp mầm tinh thể của vật liệu $\mathrm{ZnO}$ được tổng hợp và nghiên cứu một cách có hệ thống với thiết bị spin-coater tự chế tạo trên. Các kết quả phân tích cầu trúc tinh thể và hình thái học của vật liệu được đánh giá bằng phương pháp x-ray diffraction (XRD) và scanning electron microscopy (SEM).

\section{THỰC NGHIẸM}

2.1 Vật liệu và thiết bị Các vật liệu, thiết bị và linh kiện được sử dụng để chế tạo và điều khiển thiết bị spin-coater bao gồm: động cơ điện một chiều, đầu dò tốc độ quay encoder, mạch điện điều khiển động cơ L298, mạch điện tử Arduino UNO, màn hình hiển thị $\mathrm{LCD}$, bàn phím ma trận 4x4, công tắc.

Cấu tạo cơ khí của thiết bị bao gồm: đĩa quay, hộp che đĩa, khung ngoài thiết bị, chân đế cao su chống rung, hộp đựng bàn phím.

Quá trình thí nghiệm tổng hợp film mầm tinh thể $\mathrm{ZnO}$ cần sử dụng các loại hoá chất sau: Zinc acetate $\mathrm{Zn}\left(\mathrm{CH}_{3} \mathrm{COOH}\right)_{2} 99 \%$, Ethanol $\mathrm{C}_{2} \mathrm{H}_{5} \mathrm{OH} 99 \%$, Diethanol amine $\mathrm{C}_{4} \mathrm{H}_{11} \mathrm{NO}_{2} 99 \%$, tất cả hoá chất được nhập khẩu từ công ty hoá chất XiLong, Trung Quốc.

\subsection{Phương pháp}

\section{Thiết kế và chế tạo máy spin coater}

Để điều khiển tốc độ quay, trục quay của động cơ được lắp trực tiếp một tấm đĩa tròn có các vạch hấp thụ quang (334 vạch) xen kẽ, một bên có 1 đèn LED phát tia hồng ngoại xuyên qua đĩa và một bên có một đầu dò tia hồng ngoại. Thông số khoảng thời gian giữa 2 vạch đi qua đầu dò được truyền về mạch điện tử xử lý trung tâm tính toán để xác định tốc độ quay hiện tại của động cơ và sau đó sẽ phản hồi đến mạch điện điều khiển động cơ L298 tăng hoặc giảm điện áp từ 0-12 V để đạt được tốc độ mong muốn trong khoảng thời C 2020 Trường Đại học Công nghiệp thành phố Hồ Chí Minh 
gian xác định. Chương trình tính toán và vận hành thiết bị được lập trình bằng phần mềm Arduino. Các thông số tốc độ và thời gian sẽ được nhập vào bằng bàn phím ma trận $4 \times 4$.

Các bộ phận cơ khí bằng vật liệu nhựa của thiết bị được thiết kế trên phần mềm 3D Solidworks và chế tạo bằng máy in nhựa $3 \mathrm{D}$ Prusa, chất liệu nhựa PLA (là polylactic axit - nhựa nhiệt dẻo phân hủy sinh học, có nguồn gốc từ các nguồn như: bột ngô, mía, củ sắn hoặc bột khoai tây, ...thân thiện với môi trường, thường dung trong in ấn 3D) được sử dụng vì có khả năng chịu nhiệt độ cao (nóng chảy ở 180 o

C), khả năng phần huỷ sinh học cao (nguyên liệu sản xuất là bột bắp). Đặc biệt, tấm kính sẽ được cố định chắc chắn trên đĩa quay bằng 4 thanh gạc ở 4 cạnh để giữ tấm kính ở đúng tâm và không rơi ra ngoài khi thiết bị hoạt động ở tốc độ cao.

\section{Tổng hợp phim bản mỏng mầm tinh thể ZnO trên đế dẫn ITO Phương}

pháp:

Tấm kính ITO trước khi sử dụng được làm sạch bằng cách ngâm vào trong bể rửa siêu âm loại bỏ các tạp chất hữu cơ, sau đó rửa lại với ethanol và nước cất loại bỏ tạp chất vô cơ và dễ dàng bay hơi toàn bộ dung môi rửa.

Để tổng hợp được film mầm tinh thể $\mathrm{ZnO}$, cần phải có $\mathrm{Zn}(\mathrm{OH})_{2}$ sau đó nung loại bỏ $\mathrm{H}_{2} \mathrm{O}$ tạo thành $\mathrm{ZnO}$ và kết tinh trên mặt kính $\mathrm{ITO}, \mathrm{Zn}(\mathrm{OH})_{2}$ được tổng hợp bằng cách trộn đều dung dịch gồm $2.5 \times 10^{-3} \mathrm{M}$ $\mathrm{Zn}\left(\mathrm{CH}_{3} \mathrm{COO}\right)_{2}$ và 1 giọt Diethanol amine $(13 \mathrm{mg})$ - chất đóng vai trò kết dính, bằng thiết bị khuấy từ gia nhiệt với tốc độ khuấy $750 \mathrm{rpm}$, ơ $^{\circ} 40^{\circ} \mathrm{C}$ trong 15 phút.

Phủ dung dịch $\mathrm{Zn}(\mathrm{OH})_{2}$ lên kính ITO bằng phương pháp spin coating và được thực hiện trên thiết bị phủ quay được chế tạo trong đề tài này. Trước tiên, đặt 1 tấm kính ITO 20x20mm lên đĩa quay của thiết bị phủ quay và cố định chắc chắn, nhỏ 10 giọt dung dịch $\mathrm{Zn}(\mathrm{OH})_{2}$ lên tấm kính. Sau đó quay tấm kính với tốc độ quay lần 1 và lần 2 là $400 \mathrm{rpm}$ và $1500 \mathrm{rpm}$ trong khoảng thời gian là $10 \mathrm{~s}$ và $20 \mathrm{~s}$.

Tiếp theo, sấy tấm kính trong tủ sấy ở $80^{\circ} \mathrm{C}$ trong 15 phút để loại bỏ hoàn toàn dung môi, tiếp tục lặp lại quy trình phủ $\mathrm{Zn}(\mathrm{OH})_{2}$ thêm 2-3 lần với mục đích phân bố đều mầm $\mathrm{ZnO}$ trên kính ITO để kết quả tinh thể tốt nhất.

Sau cùng, nung tấm kính ITO sau khi phủ $\mathrm{ZnO}$ ở $450^{\circ} \mathrm{C}$ trong 30 phút với thiết bị nung phòng thí nghiệm và rửa nhiều lần với ethanol và nước cất. 


\section{KẾT QUẢ VÀ THẢO LUẬN}

\section{Thiết bị quay và điều khiển}

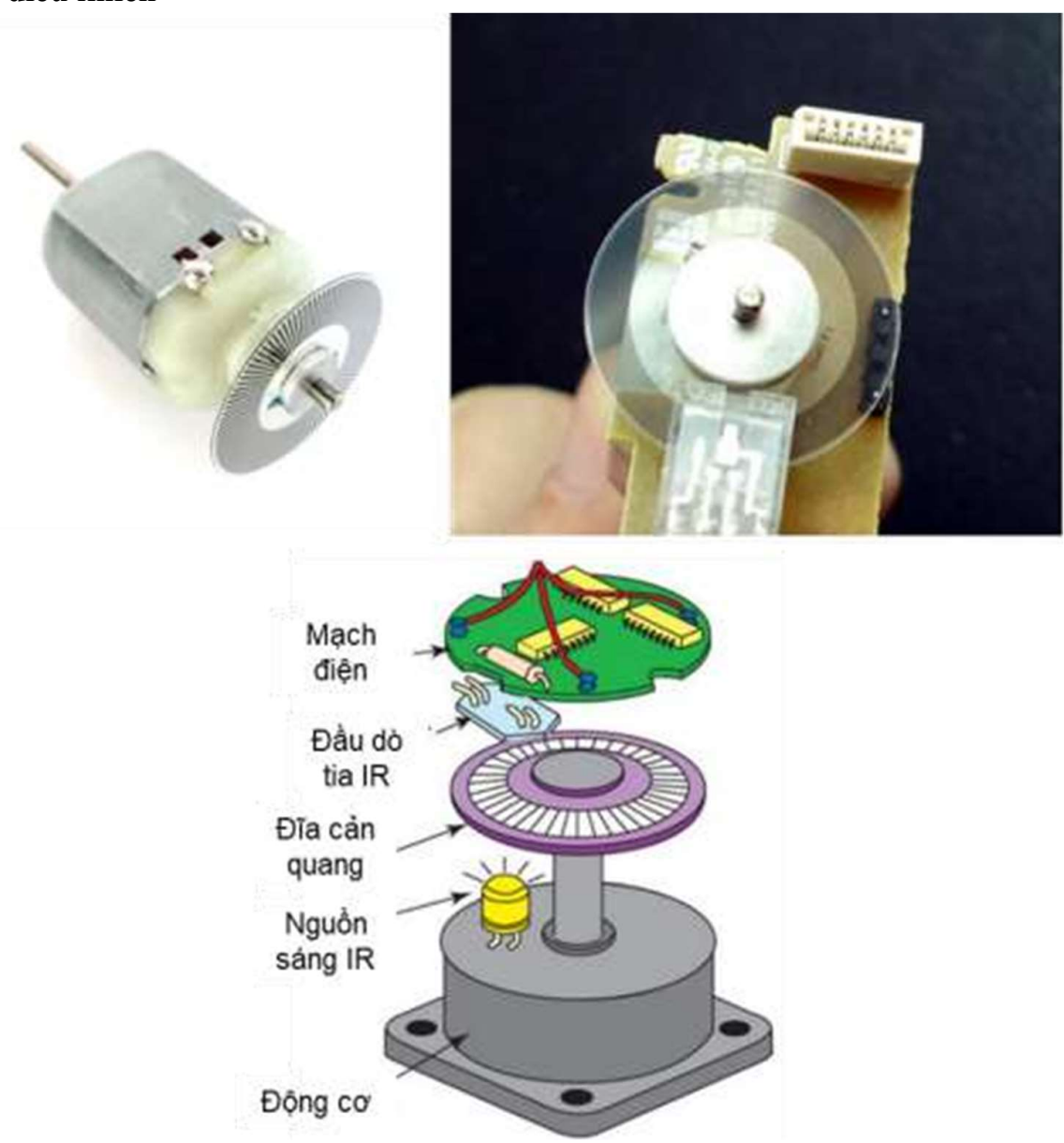

Hình 1. Hình ảnh động cơ kết hợp encoder cảm biến tốc độ quay và cơ cấu encoder

Sự kết nối giữa động cơ điện và cảm biến tốc độ quay encoder được trình bày trong sơ đồ Hình 1, trong đó đĩa cản quang được lắp trực tiếp với trục quay của động cơ, một bên LED phát ra tia hồng ngoại và một bên có bộ phận thu nhận tia hồng ngoại sau khi đi qua đĩa cản quang. Sơ đồ kết nối các linh kiện và bộ phận điện trong thiết bị spin coater, bao gồm động cơ điện một chiều, encoder, bàn phím, chip Atmega 328, module điều khiển động cơ L298, màn hình LCD. Thiết bị sử dụng nguồn điện 12 VDC được bộ biến áp chuyển đổi từ dòng điện $220 \mathrm{VAC}$ và tốc độ quay được điều chỉnh bằng cách thay đổi xung $\mathrm{PWM}$, đóng ngắt liên tục và được phản hồi liên tục bằng encoder. 

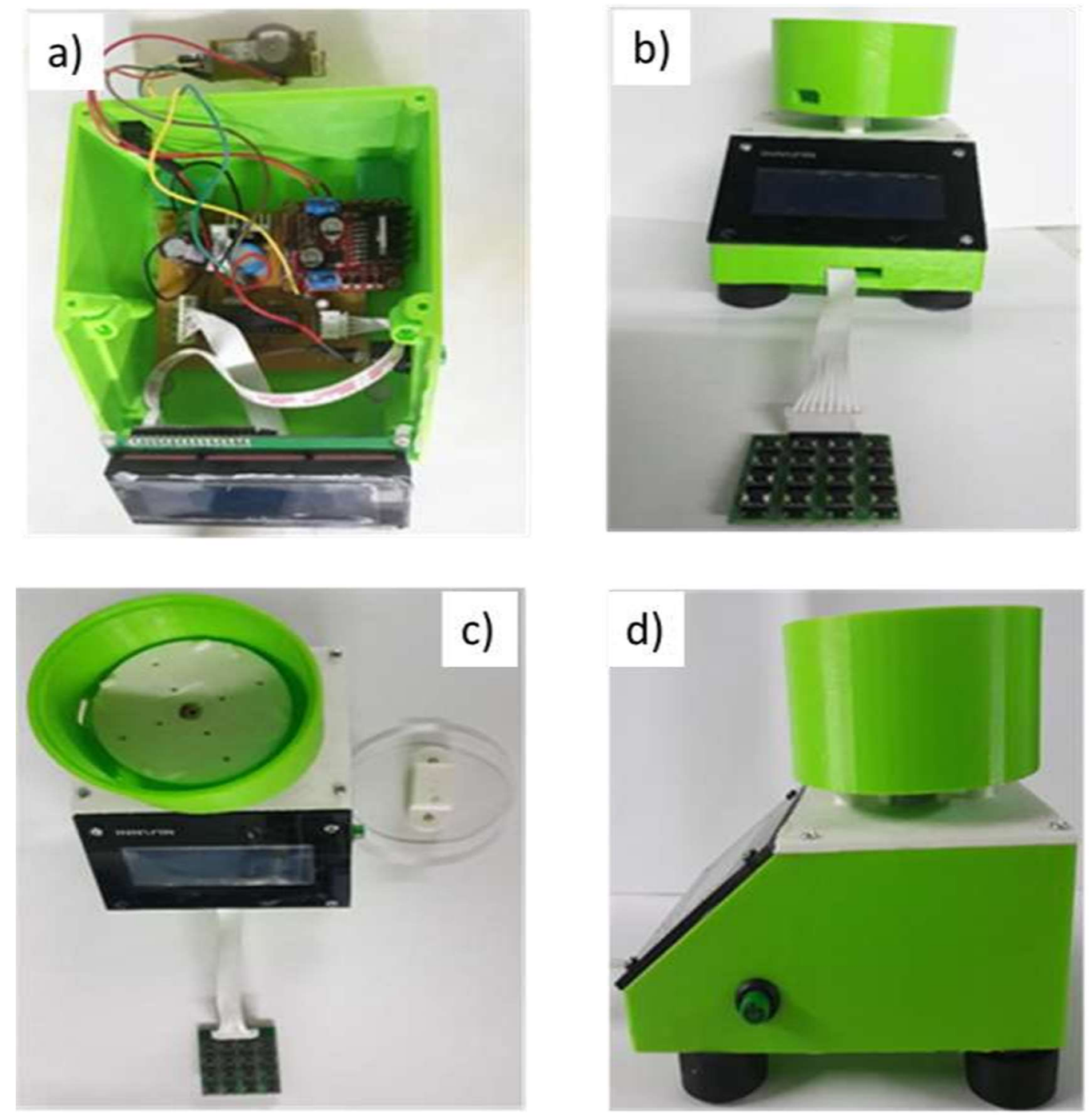

Hình 2. Khung thiết bị được chế tạo bằng công nghệ in nhựa 3D. a) Cấu tạo bên trong, b) Cấu tạo bên ngoài nhìn từ trước, c) Cấu tạo bên ngoài nhìn trừ trên, d) Cấu tạo bên ngoài nhìn từ hông

Sau khi lắp ráp linh kiện vào mạch điện, mạch điện và các bộ phận được lắp đặt vào khung nhựa được chế tạo bằng công nghệ in nhựa $3 \mathrm{D}$ được trình bày trên Hình $\mathbf{2}$. Hình $\mathbf{2 a}$ thể hiện toàn bộ các bộ phận và linh kiện điện tử được kết nối với nhau bên trong thiết bị. Hình $\mathbf{2 b}$ chụp mặt trước của máy với bàn phím hướng ra phía trước để thuận tiện thao tác. Hình $\mathbf{2 c}$ chụp mặt trên của máy cho thấy phần đĩa quay được bảo vệ bên trong trụ nhựa tròn để tránh tác động của không khí và tăng tính an toàn khi máy vận hành. Hình 2d chụp bên hông máy, nút công tắc máy được thiết bị để dễ dàng tắt máy khi xảy ra sự cố, phía dưới được lắp đệm cao su chống rung khi quay ở tốc độ cao.

Đánh giá chất lượng và hiệu quả vận hành của thiết bị thông qua quá trình tổng hợp lớp mầm tinh thể $\mathrm{ZnO}$ bằng phương pháp sol-gel. Dung dịch sol-gel cần thiết được điều chế từ Zinc acetate dehydrate $\mathrm{Zn}\left(\mathrm{CH}_{3} \mathrm{COO}\right)_{2} .2 \mathrm{H}_{2} \mathrm{O}$ hoà tan trong dung môi ethanol $\mathrm{C}_{2} \mathrm{H}_{5} \mathrm{OH}$ và diethanol amine $\mathrm{C}_{4} \mathrm{H}_{11} \mathrm{NO}_{2}(\mathrm{DEA}), \mathrm{DEA}$ không chỉ đóng vai trò chất base mà còn là chất ổn định giúp hệ phân bố đều trong thời gian dài không bị hiện tượng keo tụ. Tiền chất $\mathrm{Zn}\left(\mathrm{CH}_{3} \mathrm{COO}\right)_{2} \cdot 2 \mathrm{H}_{2} \mathrm{O}$ sau khi hòa tan vào dung dung môi, hình thành những hydroxit và sau đó được loại trừ nước bằng phương pháp nung ở nhiệt độ cao để hình thành tinh thể $\mathrm{ZnO}$. Dung dịch tiền chất được được rót phủ lên bề mặt kính dẫn điện trong suốt ITO đặt trên thiết bị spin coater một cách đồng đều và được làm bay hơi, quá trình này được lặp lại nhiều lần. 


\section{Spin Coater performance}

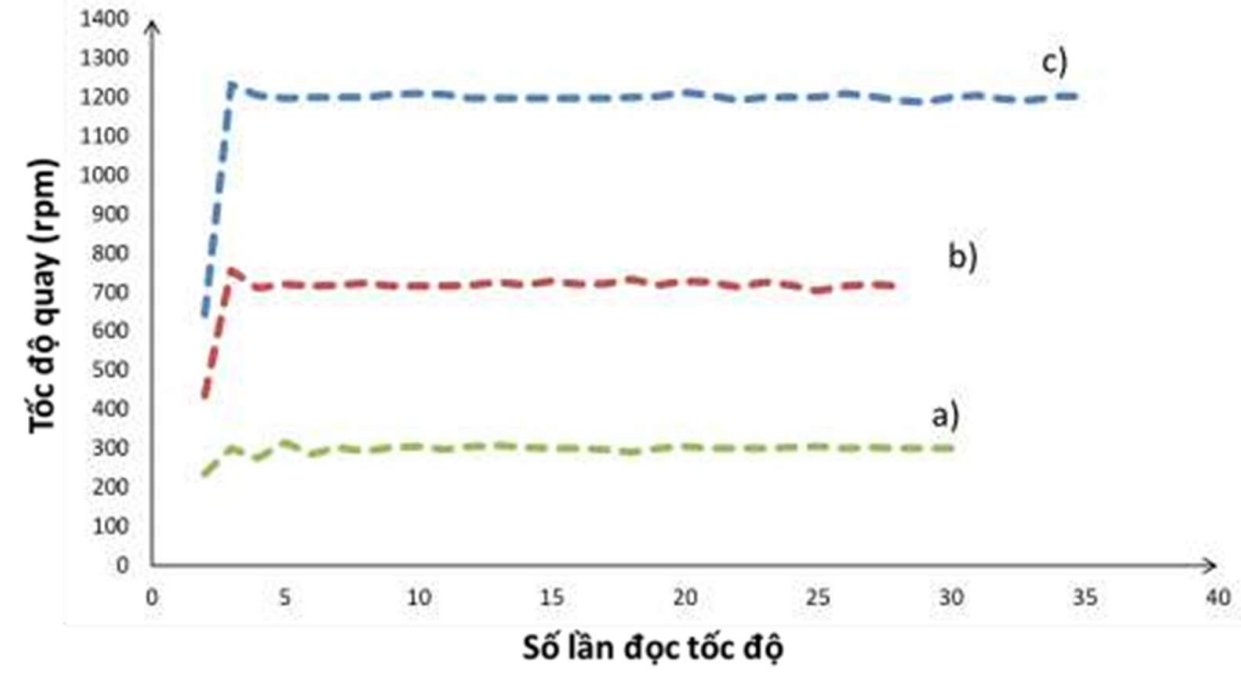

Hình 3. Khảo sát độ ổn đinh tốc độ quay thực tế của thiết bị phủ quay ( a) $300 \mathrm{rpm}$; b) $700 \mathrm{rpm}$; c) $1200 \mathrm{rpm}$ ) Quan sát Hình 3 cho thấy khi thiết bị hoạt động ở tốc độ quay thấp và cao (300 rpm, 700 rpm và $1200 \mathrm{rpm}$ ) đều đạt độ chính xác tốc độ cao (sai số tốc độ quay thực tế $3 \%<5 \%$ sai số cho phép trong kỹ thuật), độ ổn định cao theo thời gian (Biên độ dao động \pm 20 rpm).

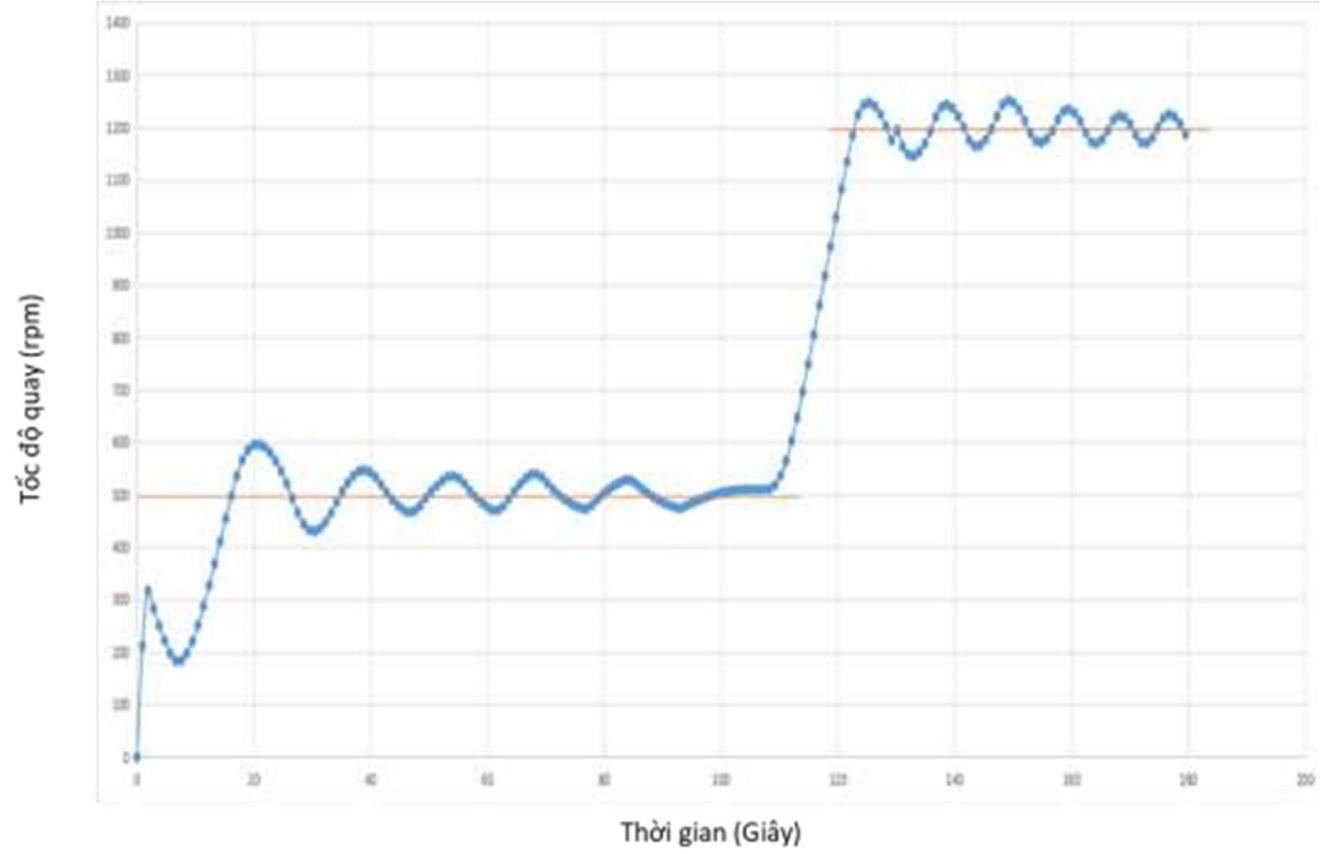

Hình 4. Khảo sát tốc độ quay thực tế của thiết bị phủ quay ở 2 pha tốc độ tuỳ chỉnh khác nhau: 500 rpm và $1200 \mathrm{rpm}$

Sự hiện diện của 2 pha tốc độ khác nhau được thể hiện qua Hình 4 khi thiết bị hoạt động ở pha tốc độ đầu và cuối là $500 \mathrm{rpm}$ và $1200 \mathrm{rpm}$. Thiết bị sẽ tự động chuyển từ pha 1 sang pha 2 sau khi hết thời gian hoạt động ở pha 1. 


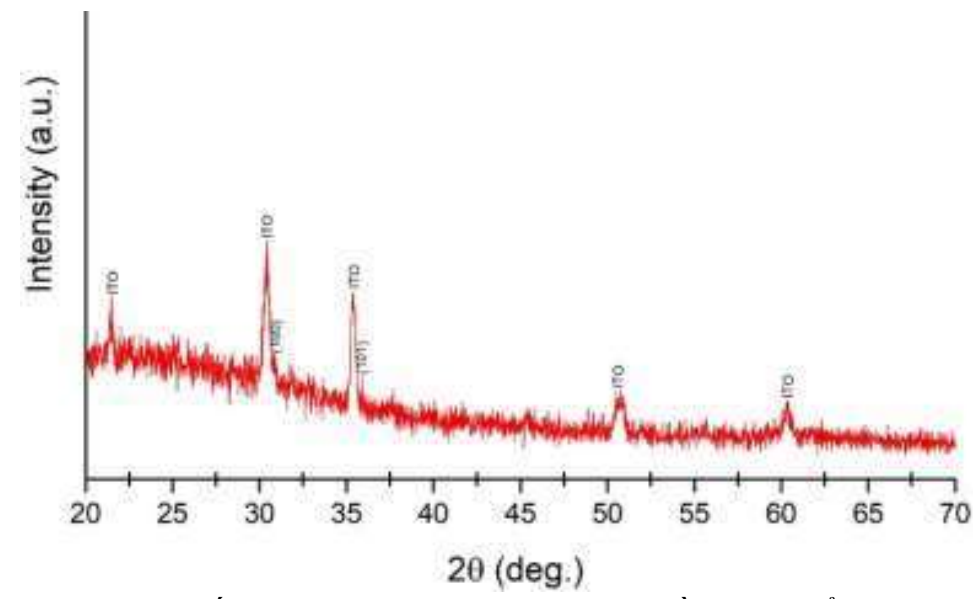

Hình 5. Kết quả XRD của film ITO phủ mầm tinh thể $\mathrm{ZnO}$

Film ZnO sau khi xử lý nhiệt để tinh thể hóa trong quy trình tổng hợp được nghiên cứu hình thái và cấu trúc tinh thể của vật liệu film. Kết quả phân tích cấu trúc tinh thể (Hình 5) qua kỹ thuật nhiễu xạ tia X (XRD) cho thấy trên bề mặt ITO xuất hiện tinh thể $\mathrm{ZnO}$ định hướng mặt (100) và (101) lần lượt tại vị trí peak $31.67^{\circ}$ và $36.14^{\circ}$, đồng thời xuất hiện peak của ITO tại $22.82^{\circ}, 30.55^{\circ}, 50.99^{\circ}$ và $60.62^{\circ}$. Ta thấy cường độ peak của ITO cao hơn so với cường độ các peak của mầm $\mathrm{ZnO}$, do lớp mầm tinh thể $\mathrm{ZnO}$ có độ dày thấp hơn nhiều so với lớp indium tin oxide trên kính dẫn ITO. Qua kết quả đã xác định được rằng thiết bị Spin Coater đã hỗ trợ trong qui trình tổng hợp được lớp mầm tinh thể $\mathrm{ZnO}$ định hướng (100) và (101), tuy nhiên, cường độ các mũi nhiễu xạ không cao và khó quan sát do kích thước hạt nhỏ và lượng ZnO không đủ để xuất hiện mũi nhiễu xạ rõ ràng.

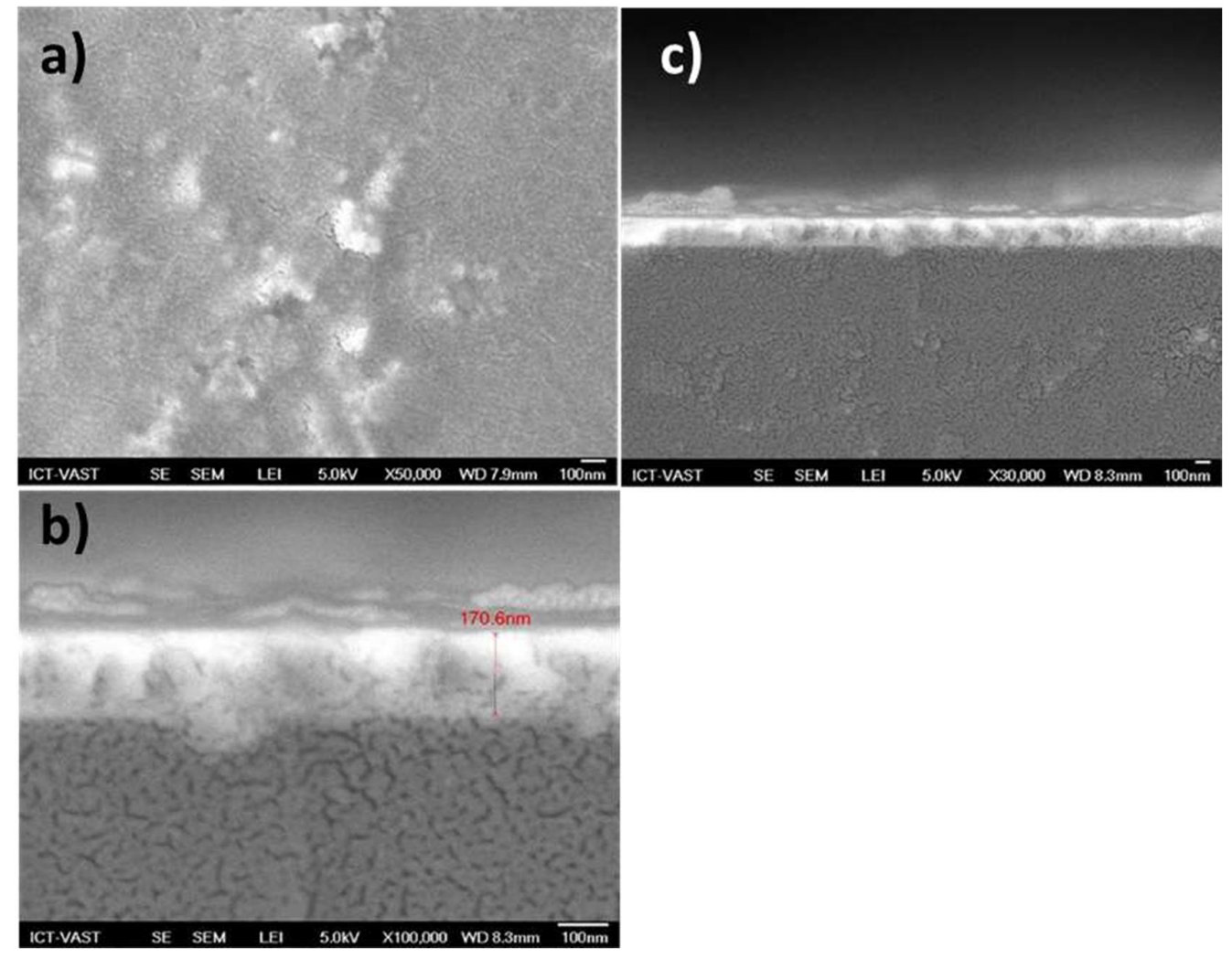

Hình 6. Hình ảnh SEM film ITO: a) Top-view bề mặt ITO chứa mầm $\mathrm{ZnO}, \mathrm{b}$ ) và c) Cross-view bề mặt ITO chứa mầm $\mathrm{ZnO}$

Hình 6 mô tả kết quả phân tích hình thái học bằng phương pháp kính hiển vi điện tử quét (SEM). Các kết quả chụp của mẫu film mầm tinh thể $\mathrm{ZnO}$ phủ trên đế dẫn ITO từ các góc chụp và độ phân giả khác 
nhau. Hình 6a) chụp góc từ trên xuống (topview) và Hình 6b), c) chụp góc từ bên cạnh (crosssection view) với các độ phân giải lớn và nhỏ. Kết quả từ hình chụp SEM cho thấy lớp cuối cùng là kính thuỷ tinh, lớp ở giữa là $\mathrm{ITO}$ có độ dày $170.6 \mathrm{~nm}$ và lớp trên cùng là mầm $\mathrm{ZnO}$ với kích thước nhỏ, khó quan sát, nằm rải rác trên bề mặt lớp ITO, tương tự với kết quả thu được từ XRD.

Để có thể tổng hợp lớp mầm $\mathrm{ZnO}$ đồng đều và có độ dày theo yêu cầu, ngoài các yếu tố như tốc độ spinning và số lần phủ, các yếu tố khác: nồng độ tiền chất phản ứng, nhiệt độ phản ứng, ... cũng ảnh hưởng lớn đến chất lượng lớp mầm $\mathrm{ZnO}$.

Sản phẩm phim bản mỏng mầm tinh thể ZnO/ITO glass
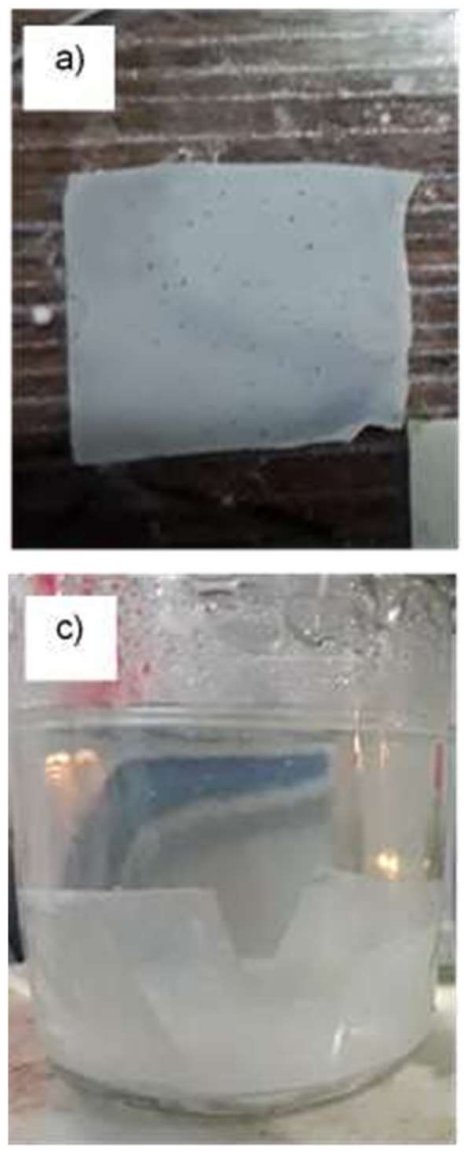
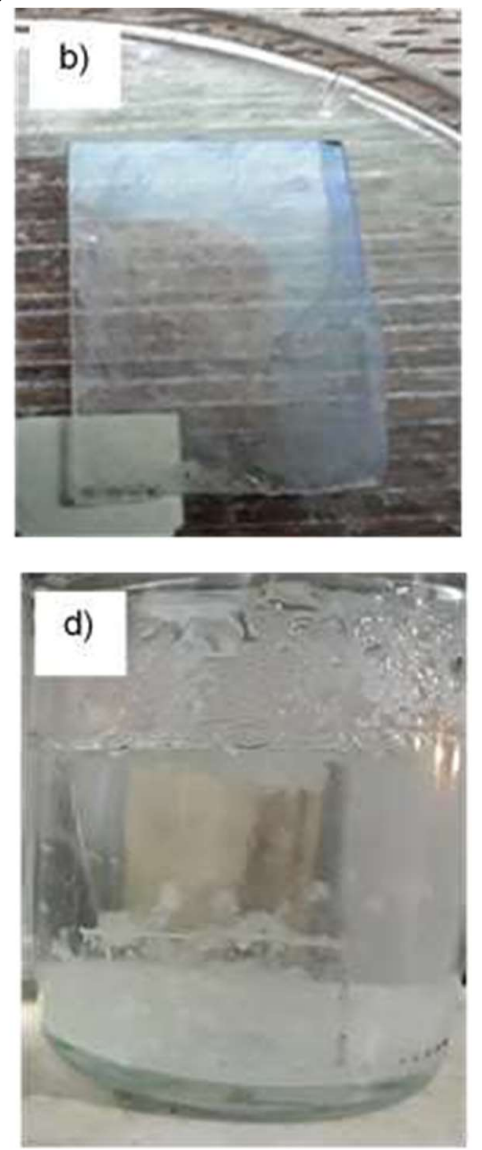

Hình 7. Hình ảnh film bản mỏng $\mathrm{ZnO}$ nanorod trong trường hợp: a) Có tạo lớp mầm $\mathrm{ZnO}$ trước khi phát triển tinh thể $\mathrm{ZnO}$ nanorod, $\mathrm{b}$ ) Không có lớp mầm $\mathrm{ZnO}$ trước khi phát triển tinh thể $\mathrm{ZnO}$ nanorod, $\mathrm{c}$ ) autolave đặt tấm kính ITO đã phủ mầm $\mathrm{ZnO}$ và $\mathrm{d}$ ) autolave đặt tấm kính ITO không phủ mầm $\mathrm{ZnO}$

Khi thực hiện quá trình thuỷ nhiệt để phát triển tinh thể $\mathrm{ZnO}$ nanorod, có thể quan sát được vùng phát triển tinh thể trong hình $\mathrm{d}$ không tập trung nhiều vào bề mặt tấm kính ITO mà thường tạo thành các mảng trắng lơ lửng hoặc bám lên mặt đáy và thành của autoclave, trong khi với hình $\mathrm{c}$ có thể thấy tinh thể $\mathrm{ZnO}$ nanorod tập trung phát triển mạnh trên bề mặt kính ITO có phủ mầm ZnO trước đó.

Kết quả film $\mathrm{ZnO}$ nanorod trong Hình $7 \mathbf{b}$ ) không đạt được màu sắc trắng đục nhiều như film $\mathrm{ZnO}$ nanorod của Hình a), được phủ mầm $\mathrm{ZnO}$.

Đối với trường hợp có lớp mầm $\mathrm{ZnO}$, tinh thể $\mathrm{ZnO}$ nanorod ưu tiên tập trung phát triển tại vị trí tâm kết tinh mầm ZnO trên kính ITO, tuy nhiên nếu không có lớp mầm $\mathrm{ZnO}$, tinh thể $\mathrm{ZnO}$ sẽ ngẫu nhiên hình thành tại bất kỳ vị trí nào không thể kiểm soát.

\section{KẾT LUẬ.}

Qua kết quả nghiên cứu, thiết bị spin coater được thiết kế và chế tạo thành công từ các vật liệu và phương pháp đơn giản, dùng phục vụ trong lĩnh vực tổng hợp vật liệu màng film mỏng với khả năng điều chỉnh được 2 pha tốc độ với độ sai số tốc độ thực tế 3\% và khoảng tốc độ điều chỉnh là 300-4800 rpm. Màng film 
bản mỏng mầm tinh thể $\mathrm{ZnO}$ được nghiên cứu tổng hợp với sự hỗ trợ của thiết bị spin coater tự chế trên. Kết quả phân tích hóa lý XRD xác định cấu trúc tinh thể $\mathrm{ZnO}$ hình thành trên lớp bán dẫn trong suốt ITO phủ trên đế kính. Hinh ảnh SEM (top view và cross-section view) cũng hỗ trợ xác nhận hình thái học và độ dày lớp film tổng hợp. Film mầm tinh thể $\mathrm{ZnO}$ là giai đoạn quan trọng để tổng hợp film tinh thể dạng que $\mathrm{ZnO}$ xếp hàng thẳng hàng trên các loại đế dẫn ITO/FTO sử dụng như các photoelectrode ứng dụng trong các phản ứng quang điện hóa được nghiên cứu và sử dụng rộng rãi hiện nay.

\section{LÒ̀I CẢM ƠN}

Kết quả nghiên cứu của đề tài được hỗ trợ bởi qũy nghiên cứu khoa học cấp trường của trường đại học Công Nghiệp TPHCM và phòng thí nghiệm, dụng cụ, thiết bị cần thiết từ khoa Công Nghệ Hoá học, trường Đại học Công nghiệp TPHCM.

\section{TÀI LIÊU THAM KHẢO}

[1] K. Norrman, Afshin Ghanbari-Siahkali, N. B. Larsen, Annu. Rep. Prog. Chem., Sect. C, 101, 2005.

[2] S. Franssila, Introduction to Microfabrication, Wiley, 2010.

[3] H. Wang, X. Yi, J. Lai \& Y. Li, International Journal of Infrared and Millimeter Waves, 26, 751-762, 2005. [4]

D. Huh, H. J. Kim, J. P. Fraser, D. E. Shea, M. Khan, A. Bahinski, G. A. Hamilton \& D. E. Ingber, Nature Protocols, 8, 2135-2157, 2013.

[5] Annu. Rep. Prog. Chem., Sect. C, 101, 174-201, 2005.

[6] M. M. Ferdaus, M. M. Rashid and M. A. Rahman, Advances in Environmental Biology, 8 (3), 729-733, 2014, [7]

P. C. Sukanek, J. Electrochem. Soc. 144, 11, 1997.

[8] W. J. Daughton, F. L. Givens, J. Electrochem. Soc. 129, 173, 1982.

[9] C. G. Rajib and P. Santanu, Chem. Rev., 112, 2373-2433, 2012.

[10] G. Liu, H. G. Yang, X. Wang, L. Cheng, J. Pan, G. Q. Lu and H-M. Cheng, J. AM. CHEM. SOC., 131, 12868$12869,2009$.

[11] H. G. Yang, C. H. Sun, S. Z. Qiao, J. Zou, G. Liu, S. C. Smith, H. M. Cheng \& G. Q. Lu, Nature, 453, 638-641, 2008.

[12] T. K. Van, L. Q. Pham, D. Y. Kim, J. Y. Zheng, D. Kim, A. U. Pawar, Y. S. Kang, ChemSusChem, 7, 3505 $3512,2014$.

[13] D. K. Bhat, Res Lett., 3:31-35, 2008.

[14] X. Wang, G. Liu, Z.-G. Chen, F. Li, L. Wang, G. Q. Lu and H.-M. Cheng, Chem. Commun., 3452-3454, 2009.

Ngày nhận bài: 02/07/2019

Ngày chấp nhận đăng: 11/01/2020 